\title{
Infinite sums associated with certain Lucas sequences
}

\author{
S. G. Rayaguru ${ }^{1}$ and G. K. Panda ${ }^{2}$ \\ ${ }^{1}$ Department of Mathematics, National Institute of Technology \\ Rourkela, India \\ e-mail: saigopalrs@gmail.com \\ ${ }^{2}$ Department of Mathematics, National Institute of Technology \\ Rourkela, India \\ e-mail: gkpanda_niterediffmail.com
}

Received: 27 May 2019

Revised: 24 December 2019

Accepted: 27 March 2020

\begin{abstract}
We obtain some closed form expressions for certain infinite sums related to Lucas and associated Lucas sequences. Some of our results generalize existing results concerning infinite sums involving Lucas sequences and others are variants of some existing results.
\end{abstract}

Keywords: Lucas sequences, Convergence of infinite series.

2010 Mathematics Subject Classification: 11B39.

\section{Introduction}

The Lucas sequence $\left(U_{n}\right)_{n \geq 1}$ and associated Lucas sequence $\left(V_{n}\right)_{n \geq 1}$ are defined by

$$
U_{n+1}=P U_{n}-Q U_{n-1}, \quad V_{n+1}=P V_{n}-Q V_{n-1},
$$

where $P, Q$ are integers such that $\Delta=P^{2}-4 Q>0$ and the initial terms are given by $\left(U_{0}, U_{1}\right)=(0,1)$ and $\left(V_{0}, V_{1}\right)=(2, P)$ respectively. The sequence $\left(U_{n}\right)_{n \geq 1}$ and $\left(V_{n}\right)_{n \geq 1}$ are often called Lucas sequence of first and second kind respectively. The Binet formulas for these sequences are given by

$$
U_{n}=\frac{\alpha^{n}-\beta^{n}}{\alpha-\beta}, V_{n}=\alpha^{n}+\beta^{n}
$$


where $\alpha, \beta=\frac{P \pm \sqrt{P^{2}-4 Q}}{2}$ are roots of the characteristic equation $X^{2}-P X+Q=0$ having discriminant $\Delta=P^{2}-4 Q$. Clearly, $\alpha+\beta=P, \alpha \beta=Q, \alpha-\beta=\sqrt{\Delta}$. These sequences can be extended to negative indices $n$ as $U_{-n}=-Q^{-n} U_{n}$ and $V_{-n}=Q^{-n} V_{n}$ respectively.

Farhi [4] obtained expressions for sums of certain infinite Lucas sequences. Some identities for the sums, ratios, products and convolutions involving some second order recurrence sequences have been explored in $[1,3,5-11]$. In the present work, we explore certain infinite sums involving Lucas and associated Lucas sequences.

\section{Preliminaries}

This section deals with some identities which will be useful for the main results of this paper.

Lemma 2.1 ([4, Lemma 2]). Let $\left(x_{n}\right)_{n \geq 1}$ be a convergent real sequence and let $x \in \mathbb{R}$ be its limit. Then, for all $k \in \mathbb{N}$, we have

$$
\sum_{n=1}^{\infty}\left(x_{n+k}-x_{n}\right)=k x-\sum_{n=1}^{k} x_{n} .
$$

The following identities can be easily proved using the Binet formulas (2) and hence, we omit the proof. Let $m, n, r$ and $t$ be integers. Then,

$$
\begin{gathered}
U_{2 n}=U_{n} V_{n}, \\
\alpha^{r} U_{n}-\alpha^{n} U_{r}=Q^{r} U_{n-r}=\beta^{r} U_{n}-\beta^{n} U_{r}, \\
\alpha^{r} V_{n}-\alpha^{n} V_{r}=-\sqrt{\Delta} Q^{r} U_{n-r}=\beta^{n} V_{r}-\beta^{r} V_{n}, \\
\alpha^{r} V_{n}-\sqrt{\Delta} \alpha^{n} U_{r}=Q^{r} V_{n-r}=\beta^{r} V_{n}+\sqrt{\Delta} \beta^{n} U_{r}, \\
U_{n+t}-\alpha^{t} U_{n}=\beta^{n} U_{t}, \\
\alpha^{t} V_{n}-\sqrt{\Delta} U_{n+t}=\beta^{n} V_{t}, \\
\alpha^{t} V_{n}-V_{n+t}=\sqrt{\Delta} \beta^{n} U_{t}, \\
\alpha^{t} U_{n} \sqrt{\Delta}+V_{n+t}=\beta^{n} V_{t}, \\
U_{n} U_{m+r}-U_{m} U_{n+r}=Q^{m} U_{r} U_{n-m}, \\
V_{n} V_{m+r}-V_{m} V_{n+r}=\Delta Q^{n} U_{r} U_{m-n}, \\
U_{n} V_{m+r}-U_{m} V_{n+r}=Q^{m} V_{r} U_{n-m}, \\
V_{n} U_{m+r}-V_{m} U_{n+r}=Q^{n} V_{r} U_{m-n}, \\
\lim _{N \rightarrow \infty} \frac{U_{N+m}}{U_{N+n}}=\lim _{N \rightarrow \infty} \frac{V_{N+m}}{V_{N+n}}=\alpha^{m-n}, \\
\lim _{N \rightarrow \infty} \frac{U_{N+m}}{V_{N+n}}=\frac{1}{\Delta} \lim _{N \rightarrow \infty} \frac{V_{N+m}}{U_{N+n}}=\frac{\alpha^{m-n}}{\sqrt{\Delta}} .
\end{gathered}
$$




\section{Main results}

In this section, we explore some infinite weighted sums with terms from Lucas and associated Lucas sequences. Some of the results of this section are variants of results of Farhi [4].

Theorem 3.1. Let $\left(a_{n}\right)_{n \geq 1}$ be a sequence of positive integers tending to infinity with $n$, and let $k$ be a positive integer. Then, for any non zero integer $t$, we have

$$
\sum_{n=1}^{\infty} Q^{a_{n}} \frac{U_{a_{n+k}-a_{n}}}{U_{a_{n+k}} U_{a_{n}}}=\frac{1}{U_{t}}\left(\sum_{n=1}^{k} \frac{U_{a_{n}+t}}{U_{a_{n}}}-k \alpha^{t}\right)
$$

Proof. In view of Eq. (11), $Q^{a_{n}} U_{t} U_{a_{n+k}-a_{n}}=U_{a_{n+k}} U_{a_{n}+t}-U_{a_{n}} U_{a_{n+k}+t}$, which implies

$$
Q^{a_{n}} \frac{U_{a_{n+k}-a_{n}}}{U_{a_{n+k}} U_{a_{n}}}=\frac{1}{U_{t}}\left(\frac{U_{a_{n}+t}}{U_{a_{n}}}-\frac{U_{a_{n+k}+t}}{U_{a_{n+k}}}\right) .
$$

Taking summation over $n$, from 1 to infinity on both sides of the above equation and then applying Lemma 2.1 for $x_{n}=\frac{U_{a_{n}+t}}{U_{a_{n}}}$, the desired result follows from Eq. (15).

Theorem 3.2. Let $\left(a_{n}\right)_{n \geq 1}$ be a sequence of positive integers tending to infinity with $n$, and let $k$ be a positive integer. Then, for any non zero integer $t$, we have

$$
\sum_{n=1}^{\infty} Q^{a_{n}} \frac{U_{a_{n+k}-a_{n}}}{U_{a_{n+k}} U_{a_{n}}}=\frac{1}{V_{t}}\left(\sum_{n=1}^{k} \frac{V_{a_{n}+t}}{U_{a_{n}}}+k \sqrt{\Delta} \alpha^{t}\right)
$$

Proof. Using Eq. (13) and (16) with $x_{n}=\frac{V_{a_{n}+t}}{U_{a_{n}}}$ in Lemma 2.1, the proof follows similar to that of Theorem 3.1.

Remark 1. Use of Eq. (7) in the right hand side of the equality in Theorem 3.1 or use of Eq. (10) in the right hand side of the equality in Theorem 3.2 gives

$$
\sum_{n=1}^{\infty} Q^{a_{n}} \frac{U_{a_{n+k}-a_{n}}}{U_{a_{n+k}} U_{a_{n}}}=\sum_{n=1}^{k} \frac{\beta^{a_{n}}}{U_{a_{n}}}
$$

which appears in [4, Theorem 1].

Corollary 3.2.1. Let $\left(a_{n}\right)_{n \geq 1}$ be an increasing arithmetic sequence of positive integers and let $r$ be its common difference. Then, for any positive integer $k$, we have

$$
\sum_{n=1}^{\infty} \frac{Q^{r(n-1)}}{U_{a_{n}} U_{a_{n+k}}}=\frac{Q^{-a_{1}}}{U_{k r}}\left(\sum_{n=1}^{k} \frac{U_{a_{n}+1}}{U_{a_{n}}}-k \alpha\right)=\frac{Q^{-a_{1}}}{P U_{k r}}\left(\sum_{n=1}^{k} \frac{V_{a_{n}+1}}{U_{a_{n}}}+k \sqrt{\Delta} \alpha\right)
$$

Proof. The result follows immediately by putting $a_{n}=r(n-1)+a_{1}$ and $t=1$ in Theorem 3.1 and Theorem 3.2 respectively.

Remark 2. Using Eq. (7) or Eq. (10) in Corollary 3.2.1 gives

$$
\sum_{n=1}^{\infty} \frac{Q^{r(n-1)}}{U_{a_{n}} U_{a_{n+k}}}=\frac{Q^{-a_{1}}}{U_{k r}} \sum_{n=1}^{k} \frac{\beta^{a_{n}}}{U_{a_{n}}},
$$

which appears in [4, Corollary 4]. 
Theorem 3.3. Let $\left(a_{n}\right)_{n \geq 1}$ be a sequence of positive integers tending to infinity with $n$, and let $k$ be a positive integer. Then, for any non zero integer $t$, we have

$$
\sum_{n=1}^{\infty} Q^{a_{n}} \frac{U_{a_{n+k}-a_{n}}}{V_{a_{n+k}} V_{a_{n}}}=\frac{1}{\Delta U_{t}}\left(k \alpha^{t}-\sum_{n=1}^{k} \frac{V_{a_{n}+t}}{V_{a_{n}}}\right)
$$

Proof. Using Eq. (12) and (15) with $x_{n}=\frac{V_{a_{n}+t}}{V_{a_{n}}}$ in Lemma 2.1, the proof follows similarly to that of Theorem 3.1.

Theorem 3.4. Let $\left(a_{n}\right)_{n \geq 1}$ be a sequence of positive integers tending to infinity with $n$, and let $k$ be a positive integer. Then, for any non zero integer $t$, we have

$$
\sum_{n=1}^{\infty} Q^{a_{n}} \frac{U_{a_{n+k}-a_{n}}}{V_{a_{n+k}} V_{a_{n}}}=\frac{1}{V_{t}}\left(\frac{k \alpha^{t}}{\sqrt{\Delta}}-\sum_{n=1}^{k} \frac{U_{a_{n}+t}}{V_{a_{n}}}\right)
$$

Proof. Using Eq. (14) and (16) with $x_{n}=\frac{U_{a_{n}+t}}{V_{a_{n}}}$ in Lemma 2.1, the proof follows similar to that of Theorem 3.3.

Theorem 3.5. Let $\left(a_{n}\right)_{n \geq 1}$ be a sequence of positive integers tending to infinity with $n$, and let $k$ be a positive integer. Then, for any non zero integer $t$, we have

$$
\sum_{n=1}^{\infty} Q^{a_{n}} \frac{U_{a_{n+k}-a_{n}}}{V_{a_{n+k}} V_{a_{n}}}=\frac{1}{\sqrt{\Delta}} \sum_{n=1}^{k} \frac{\beta^{a_{n}}}{V_{a_{n}}}
$$

Proof. The proof of this theorem follows directly by using Eq. (9) in Theorem 3.3 or using Eq. (8) in Theorem 3.4.

Corollary 3.5.1. Let $\left(a_{n}\right)_{n \geq 1}$ be a sequence of positive integers tending to infinity with $n$. Then, we have

$$
\sum_{n=1}^{\infty} Q^{a_{n}} \frac{U_{a_{n+1}-a_{n}}}{V_{a_{n+1}} V_{a_{n}}}=\frac{\beta^{a_{1}}}{\sqrt{\Delta} V_{a_{1}}} .
$$

Corollary 3.5.2. Let $\left(a_{n}\right)_{n \geq 1}$ be an increasing arithmetic sequence of positive integers and let $r$ be its common difference. Then, for any positive integer $k$, we have

$$
\sum_{n=1}^{\infty} \frac{Q^{r(n-1)}}{V_{a_{n}} V_{a_{n+k}}}=\frac{Q^{-a_{1}}}{\Delta U_{k r}}\left(k \alpha-\sum_{n=1}^{k} \frac{V_{a_{n}+1}}{V_{a_{n}}}\right)=\frac{Q^{-a_{1}}}{P U_{k r}}\left(\frac{k \alpha}{\sqrt{\Delta}}-\sum_{n=1}^{k} \frac{U_{a_{n}+1}}{V_{a_{n}}}\right)=\frac{Q^{-a_{1}}}{\sqrt{\Delta} U_{k r}} \sum_{n=1}^{k} \frac{\beta^{a_{n}}}{V_{a_{n}}}
$$

In particular,

$$
\sum_{n=1}^{\infty} \frac{Q^{r(n-1)}}{V_{a_{n}} V_{a_{n+1}}}=\frac{(\beta / Q)^{a_{1}}}{\sqrt{\Delta} U_{r} V_{a_{1}}}
$$

Proof. The first result of this corollary follows immediately by putting $u_{n}=r(n-1)+u_{1}$ and $t=1$ in Theorem 3.3, 3.4 and 3.5, while the second result of this corollary can be obtained by putting $k=1$ in the first result.

With $(P, Q)=(1,-1)$ we get the Fibonacci sequence $F_{n}=U_{n}(1,-1)$ and the Lucas sequence $L_{n}=V_{n}(1,-1)$. The following are some applications for Fibonacci and Lucas numbers: 
- Putting suitable values in Corollary 3.5.2, we get

$$
\begin{aligned}
& \sum_{n=1}^{\infty} \frac{1}{L_{2 n-1} L_{2 n+1}}=\frac{5-\sqrt{5}}{2}, \quad \sum_{n=1}^{\infty} \frac{1}{L_{2 n} L_{2 n+2}}=\frac{3 \sqrt{5}-5}{30} \\
& \sum_{n=1}^{\infty} \frac{(-1)^{n-1}}{L_{n} L_{n+1}}=\frac{5-\sqrt{5}}{2}, \quad \sum_{n=1}^{\infty} \frac{(-1)^{n-1}}{L_{n} L_{n+2}}=\frac{40-24 \sqrt{5}}{15}
\end{aligned}
$$

- Taking $a_{n}=k a^{n}(k>0, a \geq 2)$ in Corollary 3.5.1, we obtain

$$
\sum_{n=1}^{\infty} \frac{F_{(a-1) k a^{n}}}{L_{k a^{n}} L_{k a^{n+1}}}=\frac{1}{\sqrt{5} L_{k a} \Phi^{k a}}, \quad \text { where } \Phi=\frac{1+\sqrt{5}}{2} \text { is the golden ratio. }
$$

In addition, taking $a=3$ and using Eq. (3), we obtain the following formula of Bruckman and Good [2]:

$$
\sum_{n=1}^{\infty} \frac{F_{k 3^{n}}}{L_{k 3^{n+1}}}=\frac{1}{\sqrt{5} L_{3 k} \Phi^{3 k}}
$$

which gives

$$
\sum_{n=1}^{\infty} \frac{F_{3^{n}}}{L_{3^{n+1}}}=\frac{5-\sqrt{5}}{10}
$$

(which was also pointed out by Bruckman and Good [2]).

- Taking $a_{n}=F_{n}$ and $a_{n}=L_{n}$ respectively in Theorem 3.5, we obtain

$$
\sum_{n=1}^{\infty}(-1)^{F_{n}} \frac{F_{F_{n-1}}}{L_{F_{n}} L_{F_{n+1}}}=\frac{1}{4}\left(\frac{1}{\sqrt{5}}-1\right), \quad \sum_{n=1}^{\infty}(-1)^{L_{n}} \frac{F_{L_{n-1}}}{L_{L_{n}} L_{L_{n+1}}}=\frac{1}{2}\left(\frac{1}{\sqrt{5}}-\frac{1}{3}\right) .
$$

Theorem 3.6. Let $\left(a_{n}\right)_{n \geq 1}$ be a sequence of positive integers tending to infinity with $n$, and let $k$ be a positive integer. Then, we have

$$
\sum_{n=1}^{\infty}(-1)^{n} Q^{a_{n}} \frac{U_{a_{n+2 k}-a_{n}}}{V_{a_{n+2 k}} V_{a_{n}}}=-\sum_{n=1}^{k} Q^{a_{2 n-1}} \frac{U_{a_{2 n}-a_{2 n-1}}}{V_{a_{2 n}} V_{a_{2 n-1}}}
$$

Proof. The proof follows similar to that of [4, Corollary 5] by using Eq. (5).

Corollary 3.6.1. Let $\left(a_{n}\right)_{n \geq 1}$ be an increasing arithmetic sequence of positive integers and let $r$ be its common difference. Then, for any positive integer $k$, we have

$$
\sum_{n=1}^{\infty} \frac{(-1)^{n-1} Q^{r(n-1)}}{V_{a_{n}} V_{a_{n+2 k}}}=\frac{U_{r}}{U_{2 k r}} \sum_{n=1}^{k} \frac{Q^{2 r(n-1)}}{V_{a_{2 n}} V_{a_{2 n-1}}}
$$

In particular,

$$
\sum_{n=1}^{\infty} \frac{(-1)^{n-1} Q^{r(n-1)}}{V_{a_{n}} V_{a_{n+2}}}=\frac{1}{V_{a_{1}} V_{a_{2}} V_{r}}
$$

Proof. The proof is similar to that of $[4$, Corollary 6] and hence it is omitted. 
Theorem 3.7. Let $r$ and $k$ are natural numbers and $S_{r, k}=\sum_{n=1}^{\infty} \frac{(-1)^{n-1} Q^{r(n-1)}}{V_{r n} V_{r(n+k)}}$. Then for any odd positive integer $k$, we have

$$
S_{r, k}=\frac{U_{r}}{U_{r k}}\left(S_{r, 1}+Q^{r} \sum_{n=1}^{(k-1) / 2} \frac{Q^{2 r(n-1)}}{V_{2 n r} V_{(2 n+1) r}}\right)
$$

Proof. Using Eq. (13) and Corollary 3.6.1, proof of this theorem follows similarly to that of [4, Theorem 8] and hence, it is omitted.

Theorem 3.8. Let $\left(a_{n}\right)_{n \geq 1}$ be an increasing sequence of positive integers. Then, we have

$$
\sum_{n=1}^{\infty}(-1)^{n-1} \frac{\beta^{a_{n}}}{V_{a_{n}}}=\sqrt{\Delta} \sum_{n=1}^{\infty} Q^{a_{2 n-1}} \frac{V_{a_{2 n}-a_{2 n-1}}}{V_{a_{2 n}} V_{a_{2 n-1}}} .
$$

Proof. Using Eq. (5), the proof follows similar to that of [4, Theorem 11].

Theorem 3.8 can also be proved by using Eq. (15) in Theorem 3.6, when $k$ tends to infinity.

Corollary 3.8.1. If $n$ is any positive integer, then

$$
\sum_{n=1}^{\infty}\left|\frac{1}{\sqrt{\Delta}}-\frac{U_{n}}{V_{n}}\right|=\frac{2}{\sqrt{\Delta}} \sum_{n=1}^{\infty} \frac{|\beta|^{n}}{V_{n}}=2|P| \sum_{n=1}^{\infty} \frac{|Q|^{2 n-1}}{V_{2 n} V_{2 n-1}}
$$

Proof. Putting $a_{n}=n$ in Theorem 3.8, the result follows immediately using the identity $V_{n}-\sqrt{\Delta} U_{n}=2 \beta^{n}$.

Corollary 3.8.2. If $n$ is any positive integer, then

$$
\sum_{n=1}^{\infty}\left|\frac{1}{\sqrt{5}}-\frac{F_{n}}{L_{n}}\right|=\frac{2}{\sqrt{5}} \sum_{n=1}^{\infty} \frac{1}{L_{n} \Phi^{n}}=2 \sum_{n=1}^{\infty} \frac{1}{L_{2 n} L_{2 n-1}}
$$

- Further applying Theorem 3.8 for $(P, Q)=(1,-1)$ with $a_{n}=2 n, 2 n-1$ and $2 n+1$ respectively, we obtain the following summations involving Lucas numbers:

$$
\begin{aligned}
& \frac{1}{\sqrt{5}} \sum_{n=1}^{\infty} \frac{(-1)^{n-1}}{L_{2 n} \Phi^{2 n}}=\sum_{n=1}^{\infty} \frac{1}{L_{4 n} L_{4 n-2}}, \frac{1}{\sqrt{5}} \sum_{n=1}^{\infty} \frac{(-1)^{n-1}}{L_{2 n-1} \Phi^{2 n-1}}=\sum_{n=1}^{\infty} \frac{1}{L_{4 n-1} L_{4 n-3}}, \\
& \frac{1}{\sqrt{5}} \sum_{n=1}^{\infty} \frac{(-1)^{n-1}}{L_{2 n+1} \Phi^{2 n+1}}=\sum_{n=1}^{\infty} \frac{1}{L_{4 n+1} L_{4 n-1}}
\end{aligned}
$$

Theorem 3.9. If $r>1$ is any positive integer, then

$$
\sum_{n=0}^{\infty} \frac{\beta^{2^{r} n+2^{r-1}}}{V_{2^{r} n+2^{r-1}}}=\sqrt{\Delta} \sum_{n=1}^{\infty} \frac{Q^{2^{r-1} n} U_{2^{r-1} n}}{V_{2^{r} n} V_{2^{r-1} n}} .
$$

Proof. The proof is similar to that of [4, Theorem 16] using Eq. (5) and hence, we omit the proof. 


\section{Acknowledgements}

The authors are very much thankful to the anonymous referees and editor for their valuable suggestions which improved the results and presentation of the paper to a great extent.

\section{References}

[1] André-Jeannin, R. (1990). Lambert series and the summations of reciprocals in certain Fibonacci-Lucas-type sequences, Fib. Quart., 28 (3), 223-226.

[2] Bruckman, P. S., \& Good, I. J. (1976). A generalization of a series of de Morgan, with applications of Fibonacci type, Fib. Quart., 14 (3), 193-196.

[3] Davala, R. K., \& Panda, G. K. (2015). On Sum and Ratio Formulas for Balancing Numbers, Journal of the Ind. Math. Soc., 82 (1-2), 23-32.

[4] Farhi, B. (2019). Summation of Certain Infinite Lucas-Related Series, J. Int. Seq., 22 (1), Article No. 19.1.6.

[5] Frontczak, R. (2018). A Note on Hybrid Convolutions Involving Balancing and Lucasbalancing Numbers, Appl. Math. Sci., 12 (25), 2001-2008.

[6] Frontczak, R. (2018). Sums of Balancing and Lucas-balancing Numbers with Binomial Coefficients, Int. J. Math. Anal., 12 (12), 585-594.

[7] Hu, H., Sun, Z. W., \& Liu, J. X. (2001). Reciprocal sums of second order recurrent sequences, Fib. Quart., 39 (3), 214-220.

[8] Komatsu, T., \& Panda, G.K. (2018). On several kinds of sums of balancing numbers, Preprint, arXiv: $1608.05918 \mathrm{v} 3$ [math.NT].

[9] Rayaguru, S. G., \& Panda, G. K. (2018). Some Infinite Product Identities Involving Balancing and Lucas-balancing Numbers, Alabama Journal of Mathematics, 42.

[10] Rayaguru, S. G., \& Panda, G. K. (2019). Sum Formulas Involving Powers of Balancing and Lucas-balancing Numbers, Journal of the Ind. Math. Soc., 86 (1-2), 137-160.

[11] Rayaguru, S. G., \& Panda, G. K. (2019). Sum Formulas Involving Powers of Balancing and Lucas-balancing Numbers-II, Notes on Number Theory and Discrete Math., 25 (3), $102-110$. 